\title{
Recurrent subacute post-viral onset of ataxia associated with a PRF1 mutation
}

\author{
Cristina Dias ${ }^{1,2,10}$, Allison McDonald ${ }^{3,4,10}$, Murat Sincan ${ }^{5}$, Rosemarie Rupps ${ }^{1,2,6}$, Thomas Markello ${ }^{5}$, \\ Ramona Salvarinova $^{7}$, Rui F Santos ${ }^{8}$, Kamal Menghrajani ${ }^{5}$, Chidi Ahaghotu ${ }^{5}$, Darren P Sutherland ${ }^{3,4}$, \\ Edgardo S Fortuno $\mathrm{III}^{3,4}$, Tobias R Kollmann ${ }^{3,4}$, Michelle Demos ${ }^{9}$, Jan M Friedman ${ }^{1,2}$, David P Speert ${ }^{3,4}$, \\ William A Gahl ${ }^{5}$ and Cornelius F Boerkoel ${ }^{\star, 1,2,5}$
}

Inflammation is an important contributor to pediatric and adult neurodegeneration. Understanding the genetic determinants of neuroinflammation provides valuable insight into disease mechanism. We characterize a disorder of recurrent immune-mediated neurodegeneration. We report two sisters who presented with neurodegeneration triggered by infections. The proband, a previously healthy girl, presented at $\mathbf{2 2 . 5}$ months with ataxia and dysarthria following mild gastroenteritis. MRI at onset showed a symmetric signal abnormality of the cerebellar and peritrigonal white matter. Following a progressive course of partial remissions and relapses, she died at 5 years of age. Her older sister had a similar course following varicella infection, she died within 13 months. Both sisters had unremarkable routine laboratory testing, with exception of a transient mild cytopenia in the proband 19 months after presentation. Exome sequencing identified a biallelic perforin1 mutation (PRF1; p.R225W) previously associated with familial hemophagocytic lymphohistiocytosis (FHL). In contrast to FHL, these girls did not have hematopathology or cytokine overproduction. However, 3 years after disease onset, the proband had markedly deficient interleukin-1 beta (IL-1 $\beta$ ) production. These observations extend the spectrum of disease associated with perforin mutations to immune-mediated neurodegeneration triggered by infection and possibly due to primary immunodeficiency.

European Journal of Human Genetics (2013) 21, 1232-1239; doi:10.1038/ejhg.2013.20; published online 27 February 2013

Keywords: exome sequencing; neurodegeneration; cerebellar white matter; familial hemophagocytic lymphohistiocytosis; interleukin-1 beta

\section{INTRODUCTION}

Childhood neurodegeneration is a diagnostic challenge because it arises as a primary or secondary consequence of many genetic and acquired etiologies, particularly infectious causes. ${ }^{1}$ Genetic bases include disorders of metabolism, myelination, vascular integrity and inflammation. ${ }^{2,3}$ Dysfunction of the innate immune system is the most common primary inflammatory cause of neurodegeneration. ${ }^{4}$ The aberrant release of cytokines by activated microglia and circulating macrophages in disorders of the innate immune system damage the myelin sheath directly, and also enhance blood-brain barrier permeability, allowing further recruitment of leukocytes to the CNS.

The myriad of inflammatory neurodegenerative disorders rarely have pathognomonic clinical or radiographic features, and are generally subclassified as affecting primarily the white matter, the gray matter, or both. Even when specific neuroradiological signs suggest a particular subset, diagnosis remains challenging and requires additional investigations. Consequently, many neurodegenerative disorders defy a precise diagnosis.
Pro- and anti-inflammatory cytokines can both cause and mitigate neurodegeneration. ${ }^{5,6}$ Interleukin-1 beta (IL-1 $\beta$ ) is a potent proinflammatory cytokine associated with the induction and maintenance of the innate immune response. It has been implicated in neurodegeneration as well as neuroprotection (reviewed by Fogal and Hewett $^{7}$ ). IL- $1 \beta$ is overexpressed within the central nervous system of patients with multiple sclerosis, in animal models of experimental autoimmune encephalomyelitis, and systemically in some patients with active hemophagocytic lymphohistiocytosis (HLH) with CNS involvement. ${ }^{8-10}$

Familial hemophagocytic lymphohistiocytosis (FHL) is an autosomal recessive disorder of immune dysregulation, resulting in hypercytokinemia associated with fever, hepatosplenomegaly, and cytopenia. ${ }^{10,11}$ Identified genetic causes of FHL include mutations in perforin1 (PRF1; FHL2), ${ }^{12}$ UNC13D (FHL3), ${ }^{13}$ STX11 (FHL4), ${ }^{13}$ and STXBP2 (FHL5). ${ }^{14}$ The diagnostic criteria for HLH include fever, cytopenia ( $\geq 2$ cell lineages), hypertriglyceridemia and/or hypofibrinogenemia, and hemophagocytosis in the bone marrow, spleen, or lymph nodes. Additional diagnostic criteria include

${ }^{1}$ Department of Medical Genetics, University of British Columbia, Vancouver, British Columbia, Canada; ${ }^{2}$ Genetics and Health Cluster, Child and Family Research Institute, BC Children's Hospital, Vancouver, British Columbia, Canada; ${ }^{3}$ Centre for Understanding and Preventing Infection in Children, Child and Family Research Institute, Vancouver, British Columbia, Canada; ${ }^{4}$ Division of Infectious and Immunological Diseases, Department of Pediatrics, University of British Columbia, Vancouver, British Columbia, Canada; ${ }^{5} \mathrm{NIH}$ Undiagnosed Diseases Program, NIH Office of Rare Diseases Research and NHGRI, Bethesda, MD, USA; ${ }^{6}$ Rare Disease Foundation, Vancouver, British Columbia, Canada; ${ }^{7}$ Division of Biochemical Diseases, Department of Pediatrics, University of British Columbia, Vancouver, British Columbia, Canada; ${ }^{8}$ Department of Radiology, BC Children's Hospital \& University of British Columbia, Vancouver, British Columbia, Canada; ${ }^{9}$ Division of Neurology, Department of Pediatrics, University of British Columbia, Vancouver, British Columbia, Canada

${ }^{10}$ These authors contributed equally to this work

*Correspondence: Dr CF Boerkoel, Department of Medical Genetics, University of British Columbia, 4500 Oak St, Rm., C234, Vancouver, British Columbia V6H 3N1, Canada. Tel: + 604875 2157; Fax: + 604875 2376; E-mail: cornelius.boerkoel@nih.gov

Received 9 July 2012; revised 19 November 2012; accepted 23 January 2013; published online 27 February 2013 
decreased NK-cell activity, hyperferritinemia, and elevated secretion of soluble IL-2 receptor. ${ }^{15}$ Treatment includes chemo-immunotherapy and hematopoietic stem cell transplantation.

We present a family with two daughters who died of a previously undescribed neurodegenerative disorder triggered by infection. Both girls had biallelic mutations in PRF1 without diagnostic features of FHL2.

\section{MATERIALS AND METHODS}

\section{Patient data}

Clinical data on each affected individual were obtained through retrospective chart review and interviews of the parents. Data on the proposita were also collected prospectively.

Standard protocol approvals, registrations, and patient consents Family members gave informed consent/assent for protocol H09-01228 (University of British Columbia, Vancouver, Canada) and for protocol 76-HG-0238 (National Institutes of Health, Bethesda, MD, USA).

\section{Exome sequencing and analysis}

Exome sequencing was performed on the nuclear family (both affected sisters, their three unaffected siblings, and both parents - Figure 1) as previously described. ${ }^{16}$ We used NextGENe software version 2.2.2 (SoftGenetics, LLC, State College, PA, USA) to align sequences to the human reference genome (NCBI, Build 37 v2) and call variants. Each sample alignment was performed allowing one mismatched base and $\geq 85 \%$ of the read matching the reference sequence. Variant calling required that the variant was observed in $\geq 20 \%$ and $\geq 3$ short reads, with a minimum of $5 \times$ coverage.

Following generation of a variant list fitting autosomal recessive inheritance by NextGENe Viewer's Variant Comparison Tool, we ranked the variants using

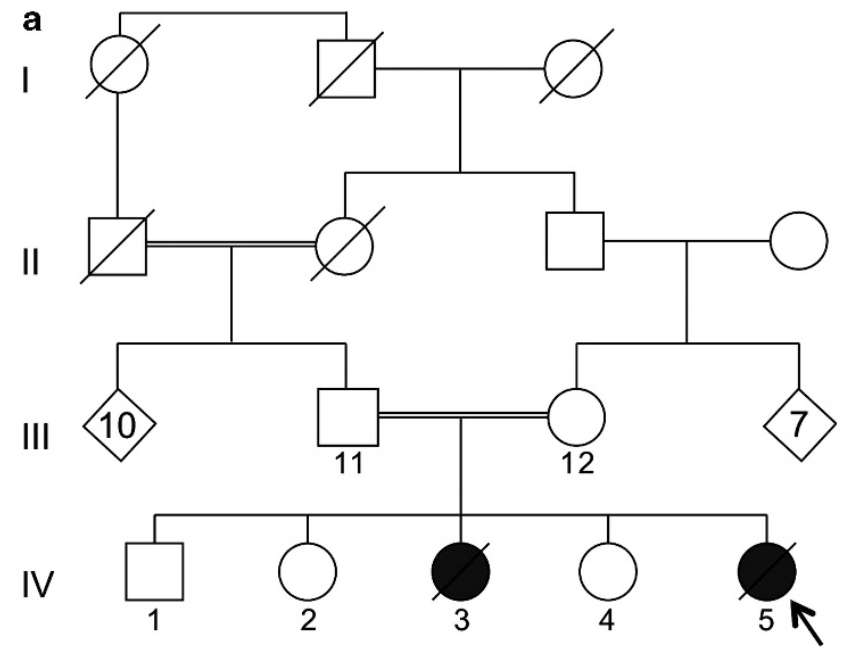

b

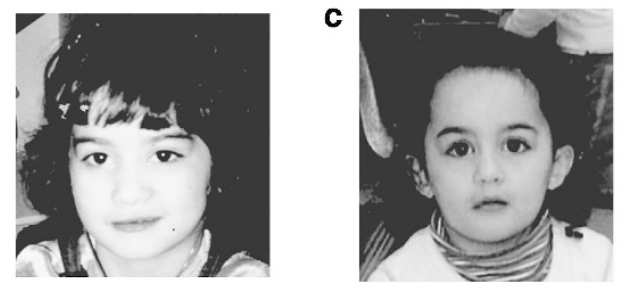

Figure 1 Familial pedigree and photographs of the affected girls. (a) The pedigree shows two loops of consanguinity; however, molecular studies and further family history analysis confirmed additional loops. The affected girls are noted by shaded shapes and the proband by an arrow. (b) Photograph of the elder affected girl. (c) Photograph of the proband.
VAR-MD as described. ${ }^{17}$ To accept variants for ranking, we required a variant to meet the following criteria in at least four of the seven exomes: a cutoff for genotype confidence score of $\geq 16$, or for variants that had scores between 7 and 16 , a genotype confidence score of at least one-fourth the coverage value.

\section{Cell stimulation and measurement of cytokines}

Peripheral blood mononuclear cells (PBMCs) were purified over a gradient of Ficoll-Paque Plus (GE Healthcare, Piscataway, NJ, USA). PBMCs were cultured in RPMI 1640 supplemented with 10\% heat-inactivated fetal calf serum and $2 \mathrm{~mm}$ L-glutamine.

For evaluation of inflammasome activation, extracellular IL-1 $\beta$ was detected in culture supernatants after stimulation of PBMCs with lipopolysaccharide (LPS) in the presence or absence of exogenous ATP. ${ }^{18}$ An LPS challenge was performed (in triplicate) on patient IV-5, unaffected siblings, parents, and seven healthy controls. Briefly, $2 \times 10^{5} \mathrm{PBMCs} /$ well were added to a 96-well plate and treated in triplicate with $10 \mathrm{ng} / \mathrm{ml}$ E. coli Ultra-Pure LPS (InvivoGen, San Diego, CA, USA) for $4 \mathrm{~h}$ with or without addition of $5 \mathrm{~mm}$ ATP for the last hour of incubation. The IL-1 $\beta$ concentrations were measured using an ELISA Ready-SET-Go (eBioscience, San Diego, CA, USA). Statistical analysis was performed using SPSS v.19 (IBM, North Castle, NY, USA), applying independent sample $t$-tests.

A Luminex-based cytokine/chemokine assessment was performed on patient and healthy adult control whole blood. ${ }^{19}$ Briefly, $2.2 \mu \mathrm{l}$ of a $10 \times$ TLR ligand for stimulation of whole blood (final working concentrations are $10 \mathrm{ng} / \mathrm{ml}$ LPS, $1 \mu \mathrm{g} / \mathrm{ml}$ PAM, $100 \mu \mathrm{g} / \mathrm{ml} \mathrm{pIC,} 10 \mu \mathrm{g} / \mathrm{ml} \mathrm{PGN}$, and $10 \mu \mathrm{M}$ R848) was added to a well of a 96-well culture plate. Assays were performed in duplicate. Two hundred microliters of a 1:1 whole blood:RPMI 1640 (Invitrogen, Carlsbad, CA, USA) mixture was then added to each well. After a 24-hour incubation, cytokine levels (IL-1 $\beta$, IL-6, IL-8, IL-10, IL-12p40, IL-12p70, IP-10, MIP-1 $\alpha$, MIP- $1 \beta$, TNF- $\alpha$, IFN- $\alpha 2$, and IFN- $\gamma$ ) were measured in the supernatants using a Luminex immunobead-based 12-plex assay (Luminex running the Masterplex software, MiraiBio, Alameda, CA, USA).

\section{RESULTS}

\section{Clinical features}

The proband and her sister (Table 1) were daughters of a consanguineous couple of Lebanese origin. The parents were first cousins but had additional loops of consanguinity such that the estimated coefficient of inbreeding for the proband was 0.13 (Supplementary data), the equivalent of the offspring of half-sibs.

Patient IV-5. The proband (IV-5, Figure 1) was born at term after an unremarkable pregnancy and delivery. She had normal early growth and development and good health aside from upper respiratory and middle ear infections. Beginning at 18 months, her weight gain declined, and at 22.5 months, a week after a bout of gastroenteritis with an eczematous rash, she developed ataxia, abnormal eye movements and dysarthria as well as brain white matter abnormalities (see below; Figure 2). She had elevated CSF protein concentration $(0.56 \mathrm{~g} / \mathrm{l}$; ref.: $0.10-0.35 \mathrm{~g} / \mathrm{l})$. Bone marrow biopsy showed iron depletion. Additional testing was otherwise unremarkable (Table 1 and Supplementary Table 1).

Soon after partial resolution of the ataxia and dysarthria, she developed progressive disease. At age 32.5 months, her brain MRI documented further white matter degeneration, and a skeletal muscle biopsy showed mild variation in fiber size and type II fiber atrophy on light microscopy; fiber and mitochondrial ultrastructure as well as mitochondrial respiratory chain activity were normal. Also, her CSF had unremarkable chemistry and cytology, and by PCR, no detectable nucleic acid indicative of infection with Cytomegalovirus, Herpes virus type 6, Varicella-Zoster, Epstein-Barr virus, Adenovirus, West Nile virus, Enterovirus, Herpes simplex viruses 1 and 2, and toxoplasmosis (Table 1). By age 42 months, she developed mild 


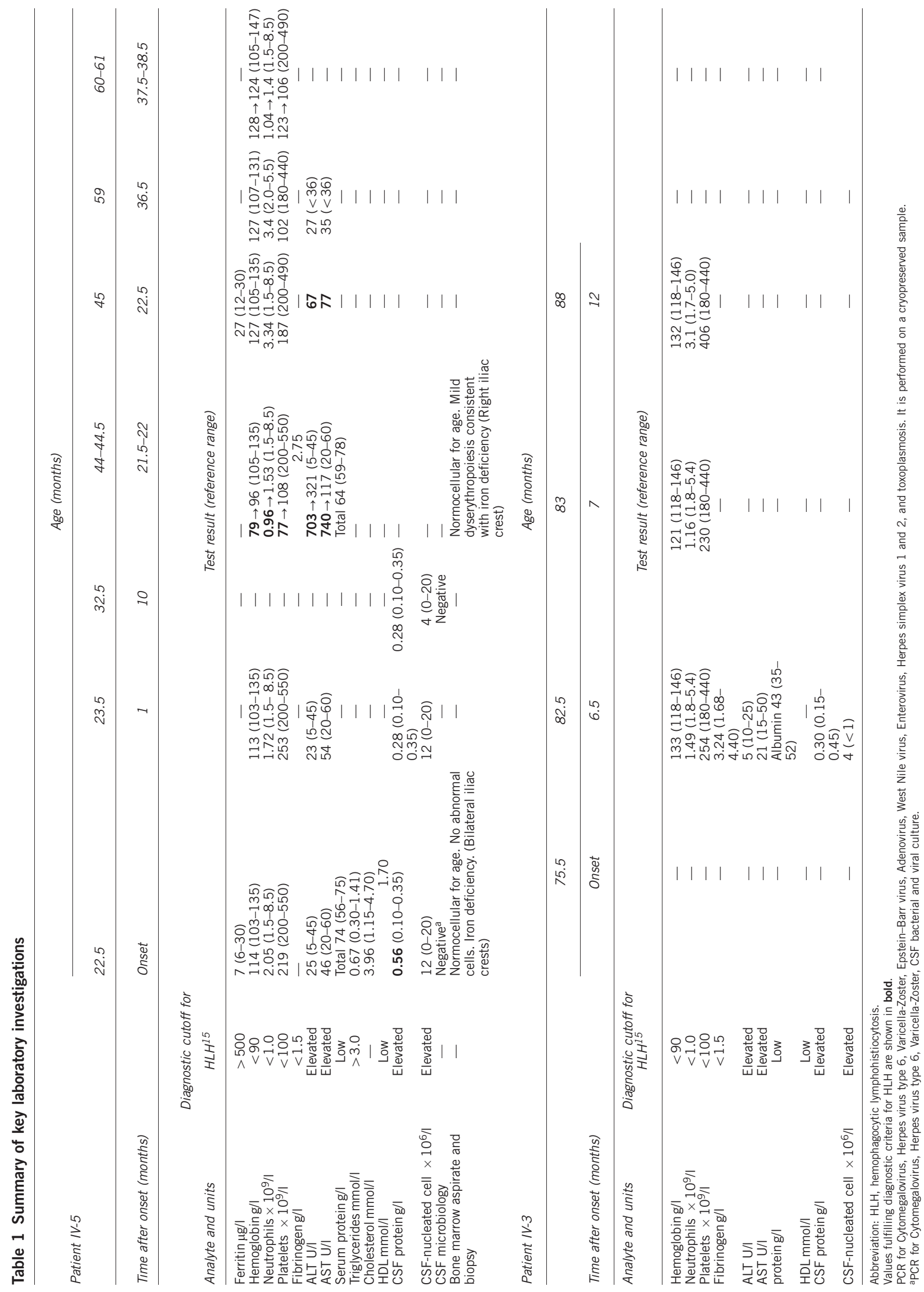



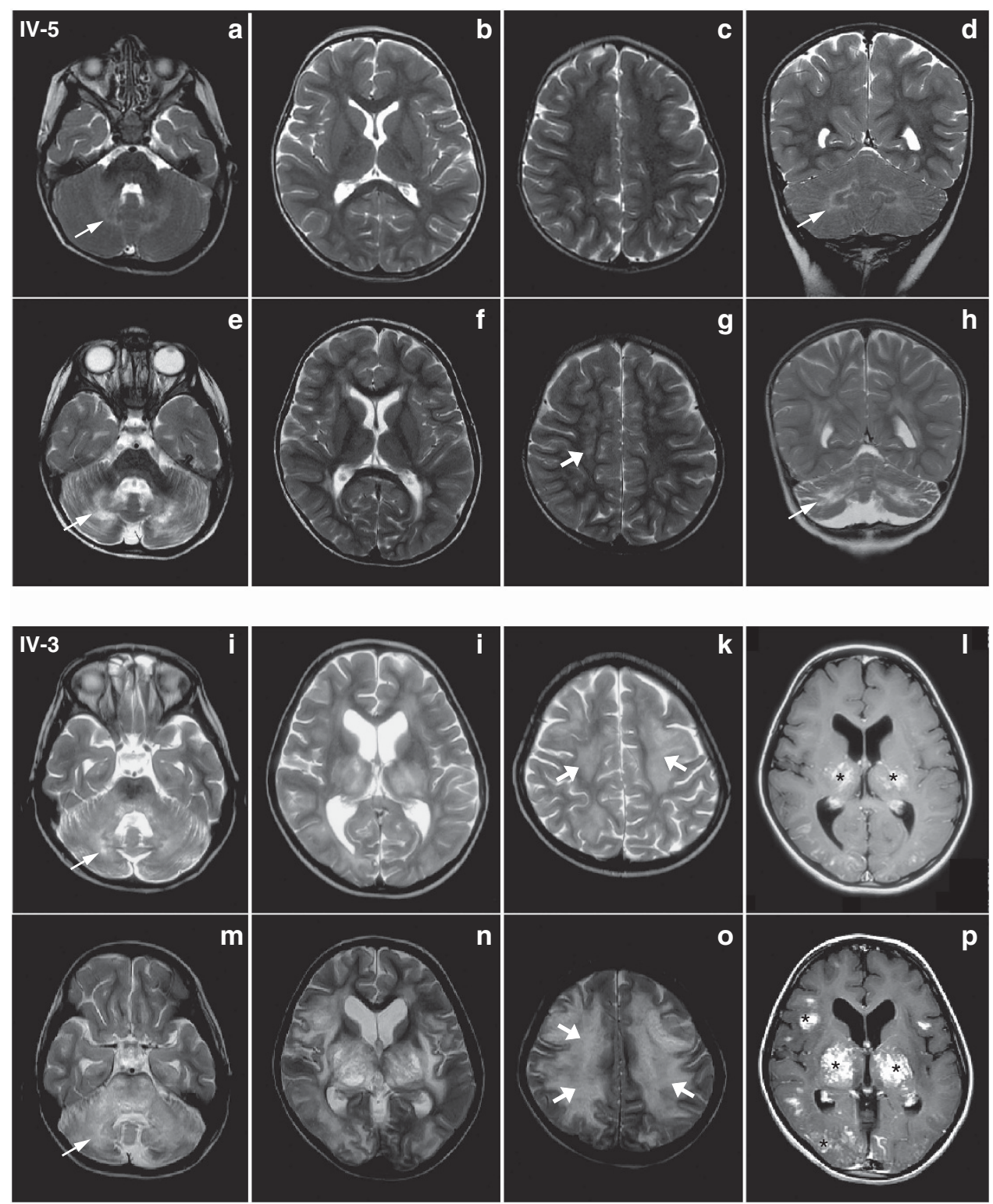

Figure 2 Brain MRI of patients IV-5 and IV-3, comparing the initial and follow-up studies. Patient IV-5: axial and coronal T2 images (a-d, 22.5 months of age, 2 weeks after onset; e-h, 32.5 months of age, 10 months after onset) show progression of signal abnormality in the cerebellar white matter (fine arrows) with atrophy of the cerebellum and subsequent involvement of the cerebral white matter (thick arrows). Patient IV-3: axial T2 and axial post-contrast T1 images (i-I, 79 months of age, 3 months after onset; $\mathbf{m}-\mathbf{p}, 88$ months of age, 12 months after onset) show extensive and progressive signal abnormality involving the cerebellar (thin arrow) and cerebral (thick arrows) white matter, thalami and basal ganglia, with some lesions showing post-contrast enhancement (asterisks).

splenomegaly and lymphadenopathy without hepatomegaly or detectable acute viral infection. Two months later, she was admitted to hospital for vomiting, tachycardia, tachypnea and fever $\left(39^{\circ} \mathrm{C}\right)$; she had hypertrophic cardiomyopathy with mild diastolic dysfunction, mild pancytopenia, and elevated AST and ALT levels. Again her bone marrow biopsy was unremarkable except for iron depletion (Table 1). Her immune studies showed normal numbers and distribution of $\mathrm{T}$, $\mathrm{B}$, and NK cells as well as unremarkable responses to mitogens and normal complement activity (Supplementary Table 1). No viral or bacterial pathogens were identified. During her recovery, she manifested persistent neck rigidity suggestive of meningeal inflammation.

Shortly after this hospitalization, she developed seizures. Over the ensuing 17 months, she gradually became blind, lost cognitive and most motor skills, and developed dysphagia. Throughout this period, she manifested neck rigidity following each of many infections but had no more recorded fevers until the week before her death when her temperature was $38.5^{\circ} \mathrm{C}$. She died at 61 months of age with upper respiratory infection and cardiac failure secondary to left ventricular hypertrophy with outlet obstruction and mitral regurgitation. The family declined an autopsy.

Patient IV-3. The proband's sister (IV-3; Figure 1) was the product of an uneventful pregnancy. She had mild developmental delay and hearing loss. At age 75.5 months, concurrent with varicella infection, she had a seizure in the context of fever, deterioration of mental status and was diagnosed with varicella meningitis. CT brain imaging 
showed dilated ventricles and cerebellar swelling. Following 2 weeks of treatment with acyclovir and steroids, she regained baseline neurological function. Subsequently, however, she became ataxic and was unable to walk. Three months after initial presentation her brain MRI showed diffuse white matter changes (Figure 2). She gradually lost additional motor skills and strength; eight months after presentation the nerve conduction velocities of her sensory and motor evoked responses were at the lower limits of normal in the right upper and lower limbs (Right Tibial Motor at ankle: latency $3.22 \mathrm{~ms}$, conduction $40.97 \mathrm{~m} / \mathrm{s}$ ). Her CSF showed normal protein, glucose and amino acid levels. CSF neurotransmitters were normal except for an elevated neopterin level; her skin fibroblasts had normal 6-pyruvoyltetrahydropterin synthase activity, excluding 6-pyruvoyltetrahydropterin synthase deficiency as the cause. All other testing was unremarkable (Table 1 and Supplementary Table 1). At age 88.5 months, her brain MRI showed progression of the white matter changes (see below; Figure 2). She died at 91 months of age, 1 year before the birth of her younger sister, the proband. No autopsy was performed.

\section{Brain imaging}

Patient IV-5. Two weeks after the onset of ataxia, a CT scan showed abnormal hyperintense areas in the cerebellar white matter. A subsequent brain MRI (Figures $2 \mathrm{a}-\mathrm{d}$ ) showed symmetrical areas of T2 hyperintense signal abnormality in the cerebellar white matter and the cerebral white matter of the frontal lobe, as well as in a peritrigonal distribution.

Ten months later a repeat brain MRI (Figure 2e-h) showed worsening of the cerebellar changes with atrophy and involvement of the gray matter. In addition, new areas of signal abnormality distributed along an anterior to posterior gradient were seen in the periventricular cerebral white matter.

Patient IV-3. Three months after her first seizure, a brain MRI (Figure 2i-1) showed cerebellar volume loss, diffuse symmetrical T1 hypointense and T2 hyperintense signal abnormalities in the cerebellar white matter, mild symmetrical ventriculomegaly and signal abnormalities in the periventricular white matter, thalami, basal ganglia, internal capsule, and midbrain. Some of these lesions enhanced after contrast, suggesting disruption of the blood-brain barrier.

Nine months later, a brain CT confirmed the prior findings, and a repeat brain MRI identified worsening of the T1 and T2 signal abnormalities involving the cerebellar white matter and extending into the gray matter, and greater involvement of the cerebral white matter, thalami, basal ganglia, internal and external capsules (Figure $2 \mathrm{~m}-\mathrm{p}$ ), and brainstem. Many lesions in the subcortical white matter, basal ganglia, cerebellar peduncles and brainstem enhanced with contrast.

\section{Defective production of IL-1 $\beta$}

In the context of her normal B- and T-cell studies, the absence of fever with apparent infection and neurodegeneration triggered by minor infections, we suspected that the proband had a defect in pyrogen generation mediated by innate immunity. To assess this, we tested if her monocytes could produce the pyrogen IL- $1 \beta$ in response to an LPS challenge in the presence of ATP (inflammasome activation assay). ${ }^{18}$ At 59 months (37 months after onset), the proband's PBMCs failed to produce IL- $1 \beta$, in contrast to healthy controls, the asymptomatic siblings, and the parents (Figure 3). Further testing using the Luminex cytokine assay confirmed the minimal IL- $1 \beta$ production and revealed a broad decrease in inflammatory cytokines,

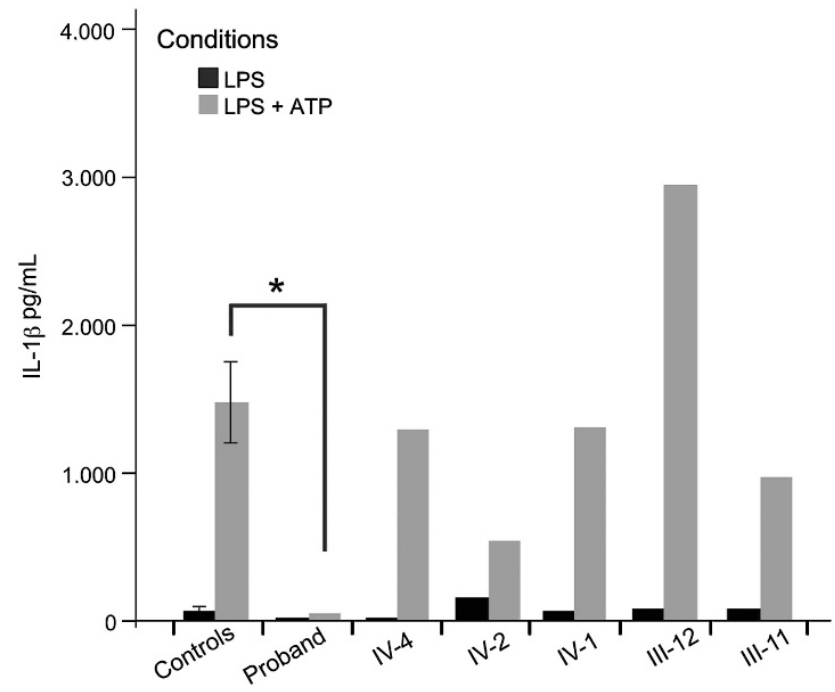

Figure 3 Inflammasome activation assay. Concentration of IL-1 $\beta$ excreted by PBMCs measured by ELISA. The cells were treated with $10 \mathrm{ng} / \mathrm{ml}$ LPS, with or without treatment with ATP. All experiments were performed in triplicate. 'Controls' represents the mean of seven biological replicates in healthy controls; error bars indicate the standard error of the mean. Proband IL-1 $\beta$ after stimulation with LPS and ATP is significantly decreased in comparison to other healthy controls, and to all five unaffected family members $(P<0.05)$. All heterozygous family members showed no significant difference from controls. Control experiments with media only, or media with ATP, yielded negligible concentration of $\mathrm{IL}-1 \beta$ in all samples (data not shown). An asterisk indicates a statistically significant difference between the patient and controls (independent samples $t$-test, $P \leq 0.05$ ).

following stimulation with a panel of innate immune activators (Supplementary Figure 1). The proband died before these abnormalities could be investigated further.

\section{Exome sequencing identifies a homozygous pathogenic PRF1 mutation}

To determine the molecular etiology for this unusual innate immune response, we performed exome sequencing on the DNA of the affected girls and immediate family members (parents and unaffected siblings; Figure 1). Analysis for homozygous variants unique to the affected siblings identified a mutation (NM_005041.4: c.673C $>$ T, p.R225W) in exon 3 of the PRF1 gene (Supplementary Figure 2). Both parents and all of the unaffected siblings were heterozygous for the same variant. The p. $\mathrm{R} 225 \mathrm{~W}$ mutation is in the transmembrane region of perforin and has been associated with FHL2. ${ }^{11,12,20,21}$ In an animal cell model, p.R225W causes decreased cytotoxicity and impaired trafficking of secretory granules. ${ }^{22}$

\section{DISCUSSION}

We present two sisters with fatal neurodegeneration showing similar patterns of white matter cerebellar degeneration. We also found that the proband had a defective innate immune response, and that both she and her affected sister had pathogenic biallelic mutations in PRF1 as a possible cause of their disease.

The PRF1 mutation identified in our patients has been associated with FHL2, ${ }^{11}$ but the sisters did not manifest diagnostic features of FHL2. ${ }^{15}$ Specifically, they had minimal or no fevers, no hypertriglyceridemia, no hypofibrinogenemia, and no hyperferritinemia. The youngest had only mild transient cytopenia. Because both sisters died before the PRF1 mutation was identified, they were not tested for 


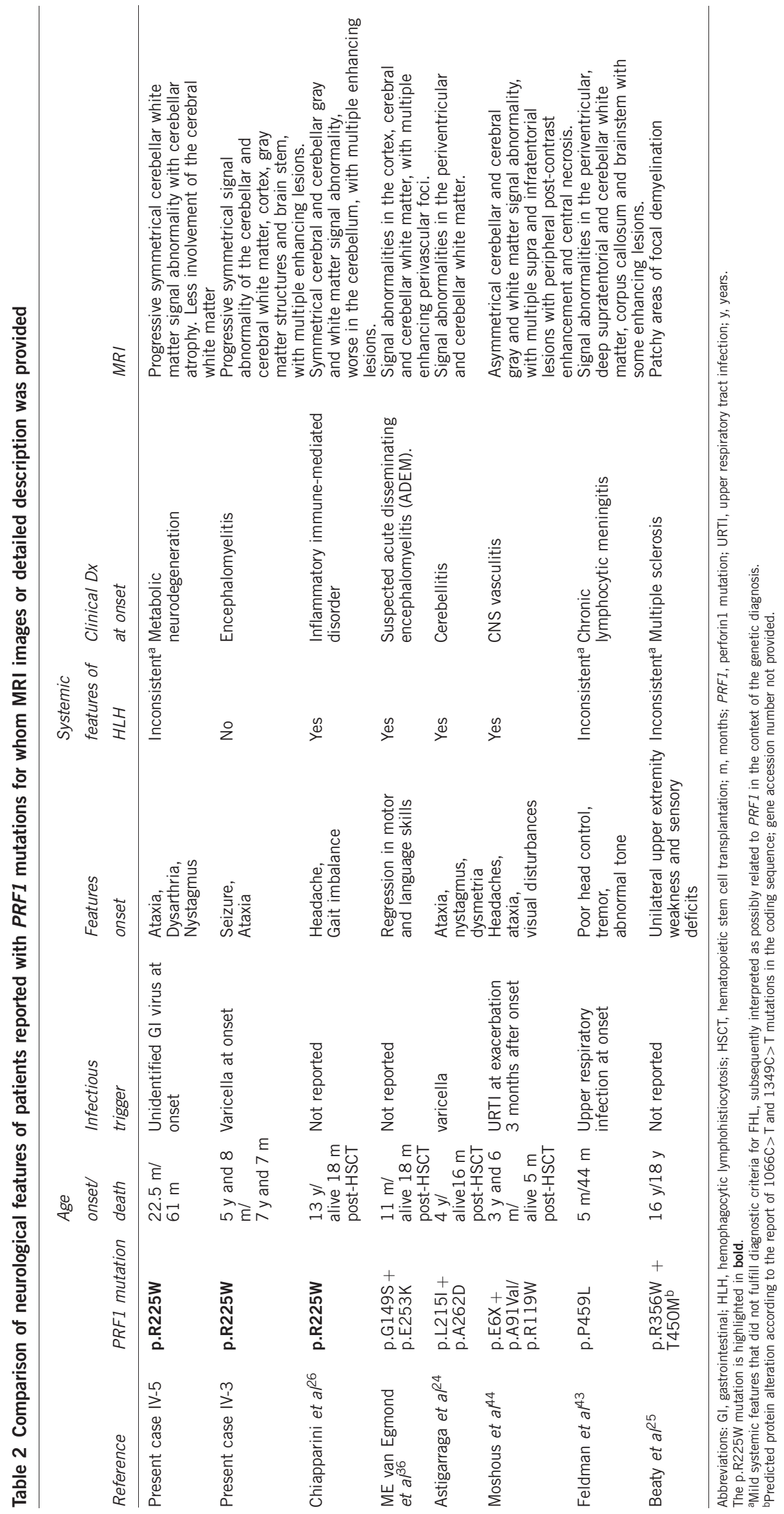


reduced NK-cell activity or elevated soluble IL-2 receptor levels. Also, two bone marrow biopsies in the proband did not demonstrate increased hemophagocytosis found in $82 \%$ of FHL2 patients. ${ }^{23}$

Neurodegeneration has been reported as a possible feature of FHL2 and sporadic $\mathrm{HLH},{ }^{24-38}$ with a frequency of $37-69 \% .^{23,29,39,40}$ A third of patients have neurological symptoms at diagnosis, ${ }^{40}$ and $36 \%$ of those with PRF1 mutations have some CNS involvement. ${ }^{23}$ Although there is an association of variations in PRF1 with susceptibility to multiple sclerosis, ${ }^{41}$ very few patients with HLH have had neurological symptoms as the sole presenting feature, and consistent with the expected ascertainment and reporting bias, ${ }^{29,30,32,39}$ all developed the diagnostic features of FHL2-HLH, with the exception of one individual who was diagnosed by brain histopathology but without

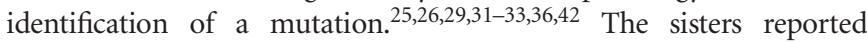
herein are therefore the first individuals reported with neurodegeneration, biallelic pathogenic PRF1 mutations and no diagnostic features suggestive of FHL-HLH (Table 2).

Potential explanations for this lack of features include the nature of the triggering pathogen, pathogen-host interaction, the immunological landscape defined by prior infectious exposures, genetic background or another primary cause of neurodegeneration. By history, however, the sisters did not have common triggering pathogens or common environmental exposures. Thus, this precludes ready acceptance of the first three possible explanations. Notwithstanding the limitations of exome sequencing, ${ }^{16}$ extensive evaluation of all exome variants in the siblings failed to identify an alternative primary causal mutation for neurodegeneration or candidate modifier variant even though the high degree of consanguinity suggests that the affected children may both have inherited such modifier variants for the phenotype associated with the PRF1 mutation (Supplementary Table 2 to 4). A genome-wide analysis of a larger cohort of patients harboring PRF1 mutations, presenting with and without neurodegeneration might, however, identify such modifiers.

In contrast to the febrile cytokine storms observed in FHL2, the proband stopped developing fevers with infection during the course of her disease. Consistent with this, her PBMCs did not produce IL- $1 \beta$ or other pro-inflammatory cytokines overexpressed in FHL2. ${ }^{10}$ This observation, consistent with a defect in the innate immune response, suggests that the link between PRF1 mutations and cytokine overexpression may be indirect or complex. The discrepant cytokine phenotype between FHL2 patients and our patients can be explained by genetic modifiers or by a difference in the timing of cytokine testing. Studies of cytokine expression in patients with classical FHL have been performed early in the disease course, most typically before treatment, ${ }^{10}$ whereas our proband was tested long after onset of symptoms. Determining whether this cytokine profile is a distinguishing feature of the PRF1-related neurodegenerative disorder manifest by these girls, or whether loss of cytokine production or cytokine depletion is a feature common to the later stages of FHL2 will require testing of additional FHL2 patients.

The brain MRI findings in HLH are nonspecific. They include multiple focal or diffuse areas of signal abnormality within the cerebral and cerebellar white and gray matter. ${ }^{28,29,36}$ The lesions can have a nodular appearance and enhance after contrast, a finding suggestive of perivascular involvement. ${ }^{29,30}$ The involvement of the cerebellum with development of cerebellar atrophy is a prominent feature observed in the majority of HLH patients. Cerebellar gray matter involvement usually appears later in the disease, and thus is not constant. Involvement of the cerebral gray matter is less common. Even when the patient cohort is limited to those with perforin mutations, the MRI findings are also not consistent (Table 2), although cerebellar disease predominates..$^{24-26,36,43,44}$ In the absence of systemic findings of $\mathrm{HLH}$, therefore, one is unable to make a clinical diagnosis of PRF1-related neurodegeneration, and molecular testing is required.

In summary, we extend the neurodegenerative phenotype associated with biallelic mutations of PRF1 and show that the neurodegenerative phenotype can occur in the absence of the hematological and immune signs of FHL2-HLH. We hypothesize that PRF1-related neurodegeneration is an under-recognized condition, and suggest that when suspicion of immune-mediated neurodegeneration arises, this diagnosis should be considered because it is potentially curable with hematopoietic stem cell transplantation. ${ }^{15,44}$

\section{WEB RESOURCES}

http://research.nhgri.nih.gov/software/Var-MD/

\section{CONFLICT OF INTEREST}

The authors declare no conflict of interest.

\section{ACKNOWLEDGEMENTS}

We are grateful to Dr Camilo Toro for manuscript review and critical analysis, and acknowledge Drs Patrick Tang and Rusang Tan for their collaboration. We would like to express a special thanks to the patients' family. This study was primarily funded by the Intramural Research Program of the NHGRI and in part by the Rare Disease Foundation. Funding was supplied by the Canadian Institutes for Health Research (DPS). DPS is the Sauder Family Professor of Pediatric Infectious Diseases. CD is supported by the Canadian Child Health Clinician Scientist Program and the Child and Family Research Institute. AM is supported by the University of British Columbia.

1 Phelan J, Lowe L, Glasier C: Pediatric neurodegenerative white matter processes leukodystrophies and beyond. Pediatr Radiol 2008; 38: 729-749.

2 Fernandes J, Saudubray J-M, Gvd Berghe, Walter JH (eds): Inborn Metabolic Diseases: Diagnosis and Treatment. Heidelberg, DEU, Germany: Springer, London, Ltd., 2006.

3 Schlüter A, Espinosa L, Fourcade $\mathrm{S}$ et al: Functional genomic analysis unravels a metabolic-inflammatory interplay in adrenoleukodystrophy. Hum Mol Genet 2011; 21 . 1062-1077.

4 Glass CK, Saijo K, Winner B, Marchetto MC, Gage FH: Mechanisms underlying inflammation in neurodegeneration. Cell 2010; 140: 918-934.

5 Amor S, Puentes F, Baker D, Van Der Valk P: Inflammation in neurodegenerative diseases. Immunology 2010; 129: 154-169

6 Bélanger M, Allaman I, Magistretti PJ: Differential effects of pro- and anti-inflammatory cytokines alone or in combinations on the metabolic profile of astrocytes. J Neurochem 2011; 116: 564-576.

7 Fogal B, Hewett SJ: Interleukin-1 $\beta$ : a bridge between inflammation and excitotoxicity? J Neurochem 2008; 106: 1-23.

8 Rossi S, Furlan R, De Chiara V et al: Interleukin-1 $\beta$ causes synaptic hyperexcitability in multiple sclerosis. Ann Neurol 2012; 71: 76-83.

9 de Jong BA, Huizinga TWJ, Bollen ELEM et al: Production of IL-1beta and IL-1Ra as risk factors for susceptibility and progression of relapse-onset multiple sclerosis. J Neuroimmunol 2002; 126: 172-179.

10 Sumegi J, Barnes MG, Nestheide SV et al: Gene expression profiling of peripheral blood mononuclear cells from children with active hemophagocytic lymphohistiocytosis. Blood 2011; 117: e151-e160.

11 Stepp SE, Dufourcq-Lagelouse Rm, Deist FoL et al: Perforin gene defects in familial hemophagocytic lymphohistiocytosis. Science 1999; 286: 1957-1959.

12 Molleran Lee S, Villanueva J, Sumegi J et al: Characterisation of diverse PRF1 mutations leading to decreased natural killer cell activity in North American families with haemophagocytic lymphohistiocytosis. J Med Genet 2004; 41: 137-144.

13 zur Stadt U, Schmidt S, Kasper B et al: Linkage of familial hemophagocytic lymphohistiocytosis (FHL) type-4 to chromosome $6 \mathrm{q} 24$ and identification of mutations in syntaxin 11. Hum Mol Genet 2005; 14: 827-834.

14 Cetica V, Santoro A, Gilmour KC et al: STXBP2 mutations in children with familial haemophagocytic lymphohistiocytosis type 5. J Med Genet 2010; 47: 595-600.

15 Henter J-I, Horne A, Aricó M et al: HLH-2004: diagnostic and therapeutic guidelines for hemophagocytic lymphohistiocytosis. Pediatr Blood Cancer 2007; 48: 124-131.

16 Dias C, Sincan M, Cherukuri PF et al: An analysis of exome sequencing for diagnostic testing of the genes associated with muscle disease and spastic paraplegia. Hum Mutat 2012; 33: 614-626. 
17 Sincan M, Simeonov D, Adams D et al: VAR-MD: A tool to analyze whole exome/ genome variants in small human pedigrees with Mendelian inheritance. Hum Mutat 2012; 33: 593-598.

18 Mehta VB, Hart J, Wewers MD: ATP-stimulated release of interleukin (IL)-1beta and IL-18 requires priming by lipopolysaccharide and is independent of caspase-1 cleavage. J Biol Chem 2001; 276: 3820-3826.

19 Kollmann TR, Crabtree J, Rein-Weston A et al: Neonatal innate TLR-mediated responses are distinct from those of adults. J Immunol 2009; 183: 7150-7160.

20 Muralitharan S, Wali YA, Dennison D et al: Novel spectrum of perforin gene mutations in familial hemophagocytic lymphohistiocytosis in ethnic omani patients. Am J Hematol 2007; 82: 1099-1102.

21 Clementi R, zur Stadt U, Savoldi G et al: Six novel mutations in the PRF1 gene in children with haemophagocytic lymphohistiocytosis. J Med Genet 2001; 38: 643-646.

22 Voskoboinik I, Thia M-C, De Bono A et al: The functional basis for hemophagocytic lymphohistiocytosis in a patient with co-inherited missense mutations in the perforin (PFN1) gene. J Exp Med 2004; 200: 811-816.

23 Trizzino A, Uz Stadt, Ueda I et al: Genotype-phenotype study of familial haemophagocytic lymphohistiocytosis due to perforin mutations. J Med Genet 2008; 45: 15-21.

24 Astigarraga I, Prats JM, Navajas A, Fernández-Teijeiro A, Urberuaga A: Near fatal cerebellar swelling in familial hemophagocytic lymphohistiocytosis. Pediatr Neurol 2004; 30: 361-364.

25 Beaty AD, Weller C, Levy B et al: A teenage boy with late onset hemophagocytic lymphohistiocytosis with predominant neurologic disease and perforin deficiency. Pediatr Blood Cancer 2008; 50: 1070-1072.

26 Chiapparini L, Uziel G, Vallinoto C et al: Hemophagocytic lymphohistiocytosis with neurological presentation: MRI findings and a nearly miss diagnosis. Neurol Sci 2011 ; 32: 473-477.

27 Chung TW: CNS Involvement in Hemophagocytic Lymphohistiocytosis: CT and MR Findings. Korean J Radiol 2007; 8: 78-81.

28 Decaminada N, Cappellini M, Mortilla M et al: Familial hemophagocytic lymphohistiocytosis: clinical and neuroradiological findings and review of the literature. Child's Nerv Syst 2010; 26: 121-127.

29 Goo H, Weon Y: A spectrum of neuroradiological findings in children with haemophagocytic lymphohistiocytosis. Pediatr Radiol 2007; 37: 1110-1117.

30 Gurgey A, Aytac S, Balta G, Oguz KK, Gumruk F: Central nervous system involvement in Turkish children with primary hemophagocytic lymphohistiocytosis. J Child Neurol 2008; 23: 1293-1299.
31 Haddad E, Sulis M-L, Jabado N, Blanche S, Fischer A, Tardieu M: Frequency and severity of central nervous system lesions in hemophagocytic lymphohistiocytosis. Blood 1997; 89: 794-800.

32 Henter J-I, Nennesmo I: Neuropathologic findings and neurologic symptoms in twenty-three children with hemophagocytic lymphohistiocytosis. J Pediatr 1997; 130: 358-365.

33 Puliyel MM, Rose W, Kumar S, Moses PD, Gibikote S: Prolonged neurologic course of familial hemophagocytic lymphohistiocytosis. Pediatr Neurol 2009; 41: 207-210.

34 Rostasy K, Kolb R, Pohl D et al: CNS disease as the main manifestation of hemophagocytic lymphohistiocytosis in two children. Neuropediatrics 2004; 35: 45-49.

35 Sieni E, Cetica V, Santoro A et al: Genotype-phenotype study of familial haemophagocytic lymphohistiocytosis type 3. J Med Genet 2011; 48: 343-352.

36 van Egmond ME, Vermeulen RJ, Peeters-Scholte CMPCD et al: Familial hemophagocytic lymphohistiocytosis in a pediatric patient diagnosed by brain magnetic resonance imaging. Neuropediatrics 2011; 42: 191-193.

37 Wada T, Nishiura K, Kuroda M et al: A case of acute encephalopathy with hemophagocytic lymphohistiocytosis and clonal T-cell expansion. Brain Dev 2011; 34: 376-379.

38 Yang S, Zhang L, Jia C, Ma H, Henter J-I, Shen K: Frequency and development of CNS involvement in Chinese children with hemophagocytic lymphohistiocytosis. Pediatr Blood Cancer 2010; 54: 408-415.

39 Deiva K, Mahlaoui N, Beaudonnet F et al: CNS involvement at the onset of primary hemophagocytic lymphohistiocytosis. Neurology 2012; 78: 1150-1156.

40 Horne A, Trottestam H, Aricò $M$ et al: Frequency and spectrum of central nervous system involvement in 193 children with haemophagocytic lymphohistiocytosis. $\mathrm{Br} \mathrm{J}$ Haematol 2008; 140: 327-335.

41 Cappellano G, Orilieri E, Comi C et al: Variations of the perforin gene in patients with multiple sclerosis. Genes Immun 2008; 9: 438-444.

42 Shinoda J, Murase S, Takenaka K, Sakai N: Isolated central nervous system hemophagocytic lymphohistiocytosis: case report. Neurosurgery 2005; 56: 187.

43 Feldmann J, Ménasché G, Callebaut I et al: Severe and progressive encephalitis as a presenting manifestation of a novel missense perforin mutation and impaired cytolytic activity. Blood 2005; 105: 2658-2663.

44 Moshous D, Feyen O, Lankisch P et al: Primary necrotizing lymphocytic central nervous system vasculitis due to perforin deficiency in a four-year-old girl. Arthritis Rheum 2007; 56: 995-999.

Supplementary Information accompanies this paper on European Journal of Human Genetics website (http://www.nature.com/ejhg) 